\title{
Systematics of intermediate-energy single-nucleon removal cross sections
}

\author{
J. A. Tostevin ${ }^{1,2}$ and A. Gade ${ }^{2,3}$ \\ ${ }^{1}$ Department of Physics, Faculty of Engineering and Physical Sciences, University of Surrey, Guildford, Surrey GU2 7XH, United Kingdom \\ ${ }^{2}$ National Superconducting Cyclotron Laboratory, Michigan State University, East Lansing, Michigan 48824, USA \\ ${ }^{3}$ Department of Physics and Astronomy, Michigan State University, East Lansing, Michigan 48824, USA
}

(Received 23 September 2014; published 26 November 2014)

\begin{abstract}
There is now a large and increasing body of experimental data and theoretical analyses for reactions that remove a single nucleon from an intermediate-energy beam of neutron- or proton-rich nuclei. In each such measurement, one obtains the inclusive cross section for the population of all bound final states of the mass $A-1$ reaction residue. These data, from different regions of the nuclear chart, and that involve weakly and strongly bound nucleons, are compared with theoretical expectations. These calculations include an approximate treatment of the reaction dynamics and shell-model descriptions of the projectile initial state, the bound final states of the residues, and the single-particle strengths computed from their overlap functions. The results are discussed in the light of recent data, more exclusive tests of the eikonal dynamical description, and calculations that take input from more microscopic nuclear structure models.
\end{abstract}

DOI: 10.1103/PhysRevC.90.057602

PACS number(s): 24.50.+g, 24.10.Ht, 25.60.-t, 25.70.-z

Fast nucleon removal reactions have been developed as an effective direct reaction, producing highly neutron-proton asymmetric nuclei with relatively high cross sections. The combination of intermediate-energy secondary beams and thick reaction targets has led to precise measurements for a large number of the most exotic nuclei. The yields are usefully large because (a) essentially all bound reaction residues are detected, with $\approx 100 \%$ efficiency, and (b) the measured cross sections are highly inclusive, with respect to the target final states. We concentrate here on the systematics of such reactions on light target nuclei, either ${ }^{9} \mathrm{Be}$ or ${ }^{12} \mathrm{C}$, for which nucleon removal associated with the Coulomb interaction (i.e., elastic Coulomb dissociation) is negligible. Here, the two strong-interaction-driven nucleon-removal mechanisms are elastic and inelastic breakup of the projectile in which the target nucleus remains in or is excited from its ground state, respectively [1].

In this work we discuss, in the main, the sum of these two contributions. In measurements that determine only the number of bound residual nuclei, the cross sections are, of course, also inclusive with respect to all bound states of the reaction residue. In such proton-neutron asymmetric systems, these final-state spectra are very often unknown or only partially known. In the following analyses these final-state spins, parities, and excitation energies are therefore normally taken from shell-model calculations and the theoretical inclusive cross sections are taken to be the sum of the calculated cross sections to all of the predicted shell-model states of the residue with excitation energies below the empirical, if known, or the evaluated [2] lowest of the particle emission thresholds of the reaction residue; that is $S_{n}$ for neutron-rich and $S_{p}$ for neutron-deficient residues.

The eikonal model theoretical description of the nucleonremoval reaction dynamics, that uses the sudden (fast collision) and eikonal (forward scattering) approximations, is presented and discussed in detail elsewhere, e.g., Refs. [1,3] and references therein. The model assumes that for the fast, surface-grazing interactions of the mass $A$ projectile with the target, of relevance to the single nucleon removal channel, the state $\alpha$ of the mass $A-1$ reaction residue is a spectator. Thus, the yield of residues in a particular final state $\alpha$ reflects a component (a parentage) of this configuration in the ground-state wave function of the projectile.

The partial cross section for removal of a nucleon, from a single-particle configuration $j^{\pi}$, populating the residue final state $\alpha$ with excitation energy $E_{\alpha}^{*}$, is calculated as

$$
\sigma_{\mathrm{th}}(\alpha)=\left(\frac{A}{A-1}\right)^{N} C^{2} S\left(\alpha, j^{\pi}\right) \sigma_{\mathrm{sp}}\left(j, S_{\alpha}^{*}\right)
$$

where $S_{\alpha}^{*}=S_{n, p}+E_{\alpha}^{*}$ is the effective separation energy for the final state $\alpha$ and $S_{n, p}$ is the ground-state to ground-state nucleon separation energy. Here $N$, in the $A$-dependent center-of-mass correction factor that multiplies the shellmodel spectroscopic factors $C^{2} S\left(\alpha, j^{\pi}\right)$, is the number of oscillator quanta associated with the major shell of the removed particle [4]. The single-particle cross section $\sigma_{\mathrm{sp}}$ is the sum of the elastic and inelastic breakup contributions to the reaction [3], $\sigma_{\mathrm{sp}}=\sigma_{\mathrm{sp}}^{\text {inel }}+\sigma_{\mathrm{sp}}^{\text {elas }}$, calculated assuming the removed-nucleon's single-particle wave function (or overlap) is normalized.

Thus, the theoretical inclusive nucleon-removal cross section, $\sigma_{\text {th }}$, is calculated as the sum of these partial cross sections $\sigma_{\text {th }}(\alpha)$ for all bound final states of the mass $A-1$ residue. This theoretical cross section is therefore an overall measure of the predicted reaction yield resulting from the single-particle strengths of the low-energy spectrum of the nucleus. There are numerous inputs to the $\sigma_{\text {th }}$ calculation (discussed below) that specify the ranges of the optical potentials and the nucleon radial overlaps and, hence, dictate the reaction geometry, and the inclusive cross section does not directly or simply probe the value of any individual spectroscopic factor, $C^{2} S\left(\alpha, j^{\pi}\right)$. The overall shell-model strengths and reaction yields can, however, be compared with the measured cross sections, $\sigma_{\text {expt }}$. This comparison is usually made in terms of the cross sections ratio $R_{s}=\sigma_{\text {expt }} / \sigma_{\text {th }}$. 
As a measure of the asymmetry of the neutron and proton binding, and that of their Fermi surfaces (that strongly affects the absolute cross sections), we use the parameter $\Delta S$. If there is just one populated final state $\alpha$, the residue ground state, then $\Delta S=S_{n}-S_{p}$ for neutron removal and $\Delta S=S_{p}-S_{n}$ for proton removal. When there are several residue final states populated then the separation energy of the removed particle in $\Delta S$ is replaced by the weighted average of their $S_{\alpha}^{*}$, each weighted by their calculated partial cross sections, $\sigma_{\text {th }}(\alpha)$. With this convention, the removal of the most strongly bound (weakly bound) nucleons from proton-neutron asymmetric nuclei have large positive (negative) values of $\Delta S$.

For each projectile, the calculation of $\sigma_{\text {th }}$ involves several inputs: (i) realistic spectra and $C^{2} S$ values, (ii) realistic residueand nucleon-target complex optical potentials and their derived elastic scattering $S$ matrices, that enter the eikonal model impact parameter integrals for $\sigma_{\mathrm{sp}}^{\text {inel }}$ and $\sigma_{\mathrm{sp}}^{\text {elas }}$ [3] and localize the reactions spatially, and (iii) realistic geometries for the radial wave functions (overlap functions) for the initial bound states of the removed nucleons in the projectile ground state. In exotic nuclei, many of these parameters are not fully constrained by experimental information. The strategy used in the analyses discussed here is to employ the best available shell-model calculations for input (i), while the shapes and radial size parameters of the optical potentials and overlaps, for inputs (ii) and (iii), are constrained by the use of Hartree-Fock (HF) calculations of neutron and proton densities for the residues and the rms radii of orbitals in the HF mean field. The procedure used, applied to all of the data sets shown here, is detailed in Ref. [5]. We note that, for most of the data sets, which are for beam energies near $100 \mathrm{MeV} /$ nucleon on a ${ }^{9} \mathrm{Be}$ target, the neutron- and proton-target potentials and their (eikonal) elastic $S$ matrices are in fact essentially common to the analyses of a large number of data sets for reactions for projectiles with a wide range of nucleon separation energies.

The first consistent analyses using this approach for data involving the removal of a well-bound nucleon, e.g., a neutron with separation energy $S_{n} \approx 22 \mathrm{MeV}$ from the proton-rich nucleus ${ }^{32} \mathrm{Ar}$ [6], now denoted ${ }^{32} \operatorname{Ar}(-n)$ with $\Delta S \approx+20 \mathrm{MeV}$, showed that the cross section ratio $R_{S}$ was unexpectedly small, with $R_{s}=0.24(3)$. Reactions involving weakly bound nucleons, on the other hand, e.g., the ${ }^{15} \mathrm{C}(-n)$ reaction with $S_{n}=1.22 \mathrm{MeV}$ and $\Delta S \approx-20 \mathrm{MeV}$, were consistent with $R_{s}$ values near unity [7]. A first systematic analysis and compilation of available data was presented in 2008, in Fig. 6 of Ref. [5]. This incorporated a previous analysis [8] of existing high-energy data for the ${ }^{12} \mathrm{C}(-n,-p)$ and ${ }^{16} \mathrm{O}(-n,-p)$ reactions, that showed consistency, for these stable nuclei, with analogous $R_{S}$ values deduced from high-energy electron-induced proton knockout. These data points, with relatively small $|\Delta S|$, are clustered near the center of Fig. 1. These suppressed $R_{S}$ values, from many electron-induced proton knockout studies on stable nuclei, have been carefully studied and quantified; see, e.g., the review of Ref. [9]. Principally, these result from nucleon singleparticle strengths in low-lying shell-model configurations being depleted due to their mixing (a) with higher-lying shells, by correlations involving the strong short-range behavior of the nucleon-nucleon interaction, and (b) with more collective

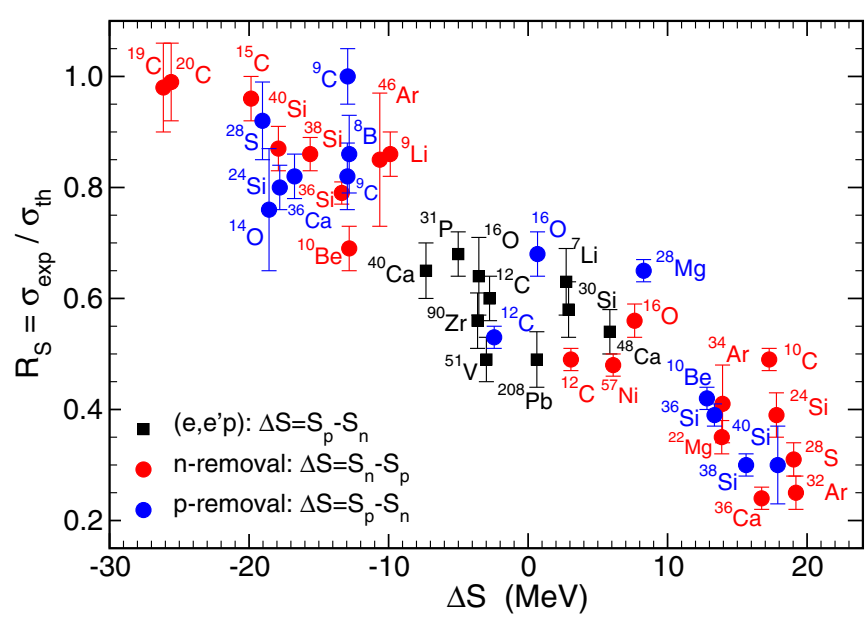

FIG. 1. (Color online) Compilation of the computed ratios $R_{s}$ of the experimental and theoretical inclusive one-nucleon-removal cross sections for each of the projectile nuclei indicated. $R_{s}$ is shown as a function of the parameter $\Delta S$, used as a measure of the asymmetry of the neutron and proton Fermi surfaces. The red points are for neutron-removal cases and the blue points those for proton removal. The solid (black) squares, deduced from electron-induced proton knockout data, are identical to the earlier compilation of Ref. [5].

configurations involving surface and/or volume correlations of longer range. Exotic beam data have allowed an exploration of the behavior of $R_{S}$ for a much-extended range of $|\Delta S|$ values away from the stable nuclei, and to include both neutron- and proton-removal reactions.

A compilation of the results of the (residue bound-statesinclusive) data and analyses, that use the common eikonal model calculations with shell-model effective interactions and model spaces appropriate to the $(N, Z)$ of the system, are shown as calculated $R_{s}=\sigma_{\text {expt }} / \sigma_{\text {th }}$ values versus $\Delta S$ in Fig. 1. Here, the reaction data shown in the earlier Fig. 6 of Ref. [5] are supplemented by the measurements and analyses for ${ }^{57} \mathrm{Ni}(-n)$ [10], ${ }^{22} \mathrm{Mg}(-n)$ [11], ${ }^{9} \mathrm{Li}(-n)$, ${ }^{9} \mathrm{C}(-p),{ }^{10} \mathrm{Be}(-n,-p),{ }^{10} \mathrm{C}(-n)[12],{ }^{36} \mathrm{Ca}(-n,-p)[13]$, ${ }^{19,20} \mathrm{C}(-n)[14],{ }^{36,38,40} \mathrm{Si}(-n,-p)[15],{ }^{28} \mathrm{Mg}(-p)[16]$, and ${ }^{14} \mathrm{O}(-p)$ [17]. The value of $R_{s}$ for this latter ${ }^{14} \mathrm{O}(-p)$ data point (measured on a carbon target), with its relatively large error bar, was recalculated here to be consistent with the HF methodology used for the other analyses. This singleparticle cross section is calculated to be $27.76 \mathrm{mb}$. So, based on the reported $\sigma_{\text {expt }}=35(5) \mathrm{mb}$, when using the ground-state to ground-state spectroscopic factor $C^{2} S=1.55$ of the Warburton-Brown two-body matrix element (TBME) shell-model Hamiltonian (WBT) (e.g., Table I of Ref. [18]), we deduce that $R_{s}=0.76(11)$, as shown in Fig. 1 . The value is smaller than, but is consistent with, the value estimated in Ref. [17].

It should also be noted that the ${ }^{10} \mathrm{Be},{ }^{36} \mathrm{Ca}$, and ${ }^{36,38,40} \mathrm{Si}$ cases, as for the earlier ${ }^{28} \mathrm{~S}(-n,-p)$ and ${ }^{24} \mathrm{Si}(-n,-p)$ data of Ref. [5], include data for the removal of nucleons of both the excess (weakly bound) and the depleted (strongly bound) species from the same projectile, with experimental (systematic uncertainty) advantages. Compared to the earlier 
compilation, Fig. 1 now includes several reactions with large positive $\Delta S$ that involve proton removal. While there is some degree of scatter on the individual points for the different projectile masses, which use shell-model analyses made with different effective interactions and/or model spaces, the trend of this large body of data is remarkably consistent. We note that the largest scatter and departures from the (nominally linear) trend, for the larger $|\Delta S|$, tend to involve the lighter nuclei studied, e.g., ${ }^{9,10} \mathrm{C}$ and ${ }^{10} \mathrm{Be}$, where individual departures from the HF- and shell-model-based calculations used are not unexpected.

Related, but more limited studies have also been made [1922] that compare the theoretical and experimental cross sections from single-nucleon pickup reactions. The work of Ref. [21], for stable nuclei, is in good agreement with the expectations of the $\left(e, e^{\prime} p\right)$ and nucleon-removal systematics shown here. From the data sets for individual transitions involving well-bound nucleons and more exotic systems the conclusions are less clear [19,20], while Ref. [22] concludes the theoretical uncertainties in simplified transfer reaction analyses can be significant.

The large majority of points in Fig. 1 are based on measurements made at the National Superconducting Cyclotron Laboratory (NSCL), at Michigan State University, with secondary beam energies in the range $80-100 \mathrm{MeV} /$ nucleon incident on a ${ }^{9} \mathrm{Be}$ target. Exceptions are the ${ }^{19,20} \mathrm{C}(-n)$ data (extreme left), measured at the Radioactive Isotope Beam Factory at RIKEN, at $240 \mathrm{MeV} /$ nucleon [14], the ${ }^{14} \mathrm{O}(-p)$ data point (with $\Delta S=-18.57 \mathrm{MeV}$ ), measured at the Heavy Ion Research Facility in Lanzhou, at $305 \mathrm{MeV} /$ nucleon [17], and the precision stable beam data from Berkeley, for the ${ }^{12} \mathrm{C}(-n,-p)$ and ${ }^{16} \mathrm{O}(-n,-p)$ reactions at 250,1050 , and $2100 \mathrm{MeV} /$ nucleon (center), as were analyzed in Ref. [8]. All of these higher energy data were measured on a carbon target. The ${ }^{10} \mathrm{C}(-n)$ and ${ }^{10} \mathrm{Be}(-n)$ reaction points (far right and left of center, respectively) were measured at the NSCL at $120 \mathrm{MeV} /$ nucleon [12].

The intermediate energy of the beam is important, generally, for the applicability of the sudden and eikonal dynamical approximations used. The beam energy is also of particular importance for the points with large positive $\Delta S$ on the far right-hand side of the figure. These involve the removal of the most strongly bound nucleons. Four-momentum conservation in an endoergic nucleon-removal reaction from the projectile will naturally impose a kinematic upper limit on the longitudinal momentum carried by the fast, forward-traveling reaction residues. As was shown dramatically in Ref. [18], where such a well-bound case, the ${ }^{14} \mathrm{O}(-n)$ reaction with $S_{n}=23.2 \mathrm{MeV}$, was performed with too low a secondary beam energy (in that case only $53 \mathrm{MeV} /$ nucleon), this kinematical cutoff intruded into and severely distorted the momentum distribution of the ${ }^{13} \mathrm{O}$ residue cross section. An expression for this maximal beam-direction residue momentum, $P_{\|}$, said to be consistent with energy and momentum conservation, was given in Eq. (1) of Ref. [18]. This was

$$
P_{\|}=\left[\left(T_{p}-S_{n}-\epsilon_{f}\right)^{2}+2 M_{r}\left(T_{p}-S_{n}-\epsilon_{f}\right)\right]^{1 / 2},
$$

where $\epsilon_{f}$ is the neutron-target relative energy in the final state. However, this expression, that makes no reference to the target mass, $M_{t}$, originates from a model that assumes $M_{t}$ is infinitely massive and should not be used for kinematics calculations with a light (e.g., $\left.{ }^{9} \mathrm{Be}\right)$ target. The correct kinematics is more complex. With $E_{i}=M_{i}+T_{i}$ and $P_{i}$ the laboratory-frame energies and momenta, and $M_{i}$ the rest energies of the projectile $(p)$, target $(t)$, reaction residue $(r)$, and removed nucleon $(N)$, and assuming a final-state excitation energy $\varepsilon_{f}^{*}$ in the removed-nucleon + target system, four-momentum conservation actually requires that

$$
\begin{aligned}
& M_{p}^{2}+M_{t}^{2}+M_{r}^{2}+2 M_{t} E_{p}-2\left(\left[E_{p}+M_{t}\right] E_{r}-P_{p} P_{\|}\right) \\
& \quad=\left(M_{t}+M_{N}+\varepsilon_{f}^{*}\right)^{2} .
\end{aligned}
$$

The general conclusion of Ref. [18] is, however, confirmed: this kinematic cutoff has minimal effect for reactions with beam energies near to and in excess of $80 \mathrm{MeV} /$ nucleon. It is also clear from Ref. [18] that any distortion observed on the high-momentum side of the (usually Gaussian-like) residue momentum distribution provides a valuable diagnostic for the onset of such effects.

For these reasons, it would certainly be of interest and value for one or more of the cases involving removal of the most well-bound nucleons to be measured at higher beam energies, say in excess of $200 \mathrm{MeV} /$ nucleon. For example, the cross section for the ${ }^{10} \mathrm{C}(-n)$ reaction at $1.6 \mathrm{GeV} /$ nucleon, with $S_{n}=21.28 \mathrm{MeV}$, was reported informally to be 21.4(17) $\mathrm{mb}$ [23]. The residue in this case, ${ }^{9} \mathrm{C}$, has only one bound state and at $1.6 \mathrm{GeV} /$ nucleon the calculated $\sigma_{\text {sp }}$ is $22.8 \mathrm{mb}$. When using the Cohen-Kurath shell-model effective interaction [24], as used and tabulated in Table V of Ref. [12], the derived $R_{S}$ is $0.49(4)$, in excellent agreement with the value $0.49(2)$ from the $120 \mathrm{MeV} /$ nucleon reaction analysis of Ref. [12]. Thus, this $1.6 \mathrm{GeV} /$ nucleon measurement coincides with and confirms the ${ }^{10} \mathrm{C}(-n)$ data point near the right-hand edge of Fig. 1. Further checks of this sort, for the $s d$ shell and heavier nuclei in this region of $\Delta S$ in the figure, would clearly be of value.

There have been several studies to try to understand the behavior of $R_{S}$ with $\Delta S$ and to test the theoretical inputs to the $\sigma_{\text {th }}$. To test the approximate description of the reaction mechanism, two recent studies probed the relative importance of the two contributions to the removal cross section as calculated using the eikonal model, that is, $\sigma_{\mathrm{sp}}^{\text {inel }}$ and $\sigma_{\mathrm{sp}}^{\text {elas }}$. These used more exclusive measurements of the final state, specifically, coincidences of the reaction residues with light charged particles. These data and analyses, that looked at both weakly bound, ${ }^{8} \mathrm{~B}(-p)$ and ${ }^{9} \mathrm{C}(-p)$ [25], and well-bound, ${ }^{28} \mathrm{Mg}(-p)$ [16], proton-removal cases, are in good agreement with the relative magnitudes of the cross sections calculated with the eikonal model.

Another approach was to try to interface the reaction dynamics with structure models that go beyond the highlytruncated-basis shell-model and effective interactions, that is, to take the residue densities, nucleon radial overlaps, and their spectroscopic factors from more microscopic calculations. In Ref. [26], this procedure was adopted for the light projectile reactions ${ }^{10} \mathrm{Be}(-n)$ and ${ }^{10} \mathrm{C}(-n)$. There, based on the no basis, variational Monte Carlo (VMC) [27] structure information, the shell-model $R_{S}$ values of Fig. 1, of 0.69(4) and 0.49(2), 
respectively, became $R_{s}^{\mathrm{VMC}}$ of $1.00(5)$ and $0.75(4)$, with significant changes from the restricted $p$-shell shell-model calculations. Because, in addition to the more extended basis, the VMC calculations include both a realistic nucleon-nucleon $(\mathrm{NN})$ interaction and a model three-nucleon $(3 \mathrm{~N})$ interaction, the most important of these physical ingredients could not be determined. In the neutron-rich oxygen isotopes, coupled-cluster calculations [28] have also pointed to the potential importance of couplings of the near-threshold neutrons to their continuum upon the spectroscopic factors of the overlaps for proton removal from the well-bound Fermi surface. However, as is shown in Eq. (1), the $C^{2} S$ are just one ingredient to $\sigma_{\text {th }}$ and $R_{s}$ and the spatial extent and the radial forms of these proton overlaps, and also the residue densities, are required for a consistent calculation of these effects upon the removal reaction cross sections. All of these ingredients and comparative cross section calculations should be possible soon.

Such efforts have concentrated on the suppressed $R_{S}$ values for large positive $\Delta S$ in the figure. There has been very little discussion of the larger $R_{S}$ with large negative $\Delta S$, where it is more natural to associate the weak binding and larger radial extent of the nucleon orbitals with reduced correlation effects beyond the shell model. This is in keeping with the trends observed of the coupled-cluster calculations of Ref. [28].

In this Brief Report we have brought up to date a comparison of measured and calculated inclusive one-nucleon-removal cross sections, in the light of analyses of a large body of new experimental data. Recent more exclusive measurements have also confirmed the calculated fractional contributions to the reaction from the elastic and inelastic nucleon-removal mechanisms, adding confidence to this aspect of the theoretical predictions. The new body of data conforms to the earlier trends of the ratio of the measured and theoretical cross sections, $R_{s}=\sigma_{\text {expt }} / \sigma_{\text {th }}$, with the neutron-proton separation energy asymmetry parameter $\Delta S$. Recent theoretical results, based on structure models that go beyond the truncated-basis, configuration-interaction shell model, suggest that extended bases, 3N force effects, and explicit couplings of nearthreshold single-particle configurations to the continuum may all play a role in understanding the observed dependence of $R_{s}$ on $\Delta S$. The present eikonal reaction plus shell-model structure theory approach predicts that more single-particle strength and nucleon-removal cross section leads to bound final states of the residues than is observed, in both neutron and proton removal, particularly in reactions that remove very well-bound nucleons of the deficient species. The new data for weakly bound neutron and proton removals with large negative $\Delta S$ have $R_{S}$ values consistent with unity, with little evidence of the need for any significant correlation effects beyond the shell model.

This work was supported by the National Science Foundation under Grant No. PHY-1102511 and the United Kingdom Science and Technology Facilities Council (STFC) under Grants No. ST/J000051/1 and No. ST/L005743/1.
[1] P. G. Hansen and J. A. Tostevin, Annu. Rev. Nucl. Part. Sci. 53, 219 (2003).

[2] G. Audi, A. H. Wapstra, and C. Thibault, Nucl. Phys. A 729, 337 (2003).

[3] J. A. Tostevin, Nucl. Phys. A 682, 320 (2001).

[4] A. E. L. Dieperink and T. L. de Forest, Jr., Phys. Rev. C 10, 543 (1974).

[5] A. Gade et al., Phys. Rev. C 77, 044306 (2008).

[6] A. Gade et al., Phys. Rev. Lett. 93, 042501 (2004).

[7] J. R. Terry et al., Phys. Rev. C 69, 054306 (2004).

[8] B. A. Brown, P. G. Hansen, B. M. Sherrill, and J. A. Tostevin, Phys. Rev. C 65, 061601(R) (2002).

[9] W. Dickhoff and C. Barbieri, Prog. Part. Nucl. Phys. 52, 377 (2004).

[10] K. L. Yurkewicz et al., Phys. Rev. C 74, 024304 (2006).

[11] C. Aa. Diget et al., Phys. Rev. C 77, 064309 (2008).

[12] G. F. Grinyer et al., Phys. Rev. C 86, 024315 (2012).

[13] R. Shane et al., Phys. Rev. C 85, 064612 (2012).

[14] N. Kobayashi et al., Phys. Rev. C 86, 054604 (2012).

[15] S. R. Stroberg et al., Phys. Rev. C 90, 034301 (2014).

[16] K. Wimmer et al., Phys. Rev. C (to be published).
[17] Z. Y. Sun et al., Phys. Rev. C 90, 037601 (2014).

[18] F. Flavigny, A. Obertelli, A. Bonaccorso, G. F. Grinyer, C. Louchart, L. Nalpas, and A. Signoracci, Phys. Rev. Lett. 108, 252501 (2012).

[19] Jenny Lee et al., Phys. Rev. Lett. 104, 112701 (2010).

[20] F. Flavigny et al., Phys. Rev. Lett. 110, 122503 (2013).

[21] B. P. Kay, J. P. Schiffer, and S. J. Freeman, Phys. Rev. Lett. 111, 042502 (2013).

[22] F. M. Nunes, A. Deltuva, and June Hong, Phys. Rev. C 83, 034610 (2011).

[23] V. Volkov et al., Helmholtzzentrum für Schwerionenforschung GmbH, GSI Report No. PHN-NUSTAR-FRS-09, 2011, p. 151 (unpublished).

[24] S. Cohen and D. Kurath, Nucl. Phys. 73, 1 (1965).

[25] D. Bazin et al., Phys. Rev. Lett. 102, 232501 (2009).

[26] G. F. Grinyer et al., Phys. Rev. Lett. 106, 162502 (2011).

[27] S. C. Pieper and R. B. Wiringa, Annu. Rev. Nucl. Part. Sci. 51, 53 (2001).

[28] Ø. Jensen, G. Hagen, M. Hjorth-Jensen, B. A. Brown, and A. Gade, Phys. Rev. Lett. 107, 032501 (2011). 